\title{
Idiopathic scrotal calcinosis. A case report
}

\author{
\begin{tabular}{c}
\hline Ionica-Mariana Radulescu",2, Ana Arghir1, Cosmin Moldovan'1 \\
${ }^{1}$ Faculty of Medicine, "Titu Maiorescu" University of Bucharest, Bucharest, Romania \\
${ }^{2}$ Regina Maria Private Health Network, "Titu Maiorescu" Policlinic, Bucharest, Romania \\
\hline
\end{tabular}
}

\begin{abstract}
Idiopathic scrotal calcinosis (ISC) is a benign, rare condition with incompletely elucidated etiology. It is clinically characterized by the presence of multiple calcified, asymptomatic nodules, located in the scrotum. The onset of the disease occurs during childhood or young adulthood. We present the case of a patient with idiopathic scrotal calcinosis with onset around the age of 20 years. The peculiarity of the case lies in the rarity of this condition and the fact that the patient came to our clinic about 25 years after the onset of his condition, even though he had multiple nodules, which makes it difficult to diagnose and complicates the promptness of surgery.
\end{abstract}

Keywords: calcifications, calcinosis, idiopathic, scrotum

\section{INTRODUCTION}

Idiopathic scrotal calcinosis (ISC) is part of a group of conditions characterized by the presence of dermic or hypodermic deposits of calcium hydroxyapatite crystals or amorphous calcium phosphate, called calcinosis cutis (1-3).

Idiopathic calcinosis, unlike other types of calcinosis cutis (dystrophic, metastatic, iatrogenic, calciphylaxis) is not associated with damage to the underlying tissue and abnormal levels of calcium or phosphorus $(4,5)$.

Idiopathic calcinosis can be classified in localized calcinosis, that include scrotal calcinosis, Winer's solitary nodular calcinoma and calcinosis circumscripta, and generalized calcinosis or calcinosis universalis $(6,7)$.

\section{CASE PRESENTATION}

We present the case of a 45-year-old patient, who lives in an urban environment, presented himself for physical examination for multiple nodular formations, located in the scrotum, without any subjective signs. The lesions developed at the age of 20. There were no similar injuries in the family history, and his personal medical history was of no significance, there was no record of trauma or infections in the scrotum. Also, he had no systemic or metabolic disease.

At the dermatological examination, multiple, firm, painless, white-yellow nodules were observed, with variable sizes between 0.5 and 2.5 $\mathrm{cm}$, located in the scrotum (Figure 1).

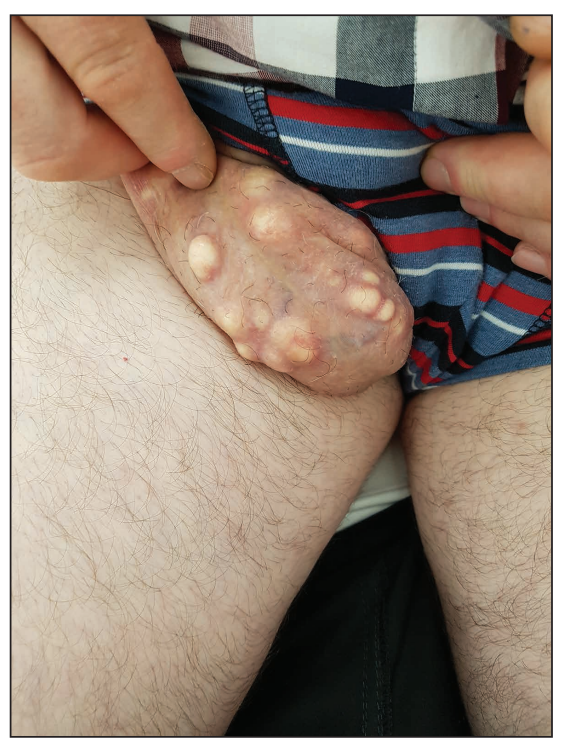

FIGURE 1. Multiple, firm, yellow, skin nodules, with dimensions between 0.5 and $2 \mathrm{~cm}$, located on the scrotum 
The testes were normal, and there was no inguinal lymphadenopathy. The nodules had progressively increased in size and number over time (Figure 2).

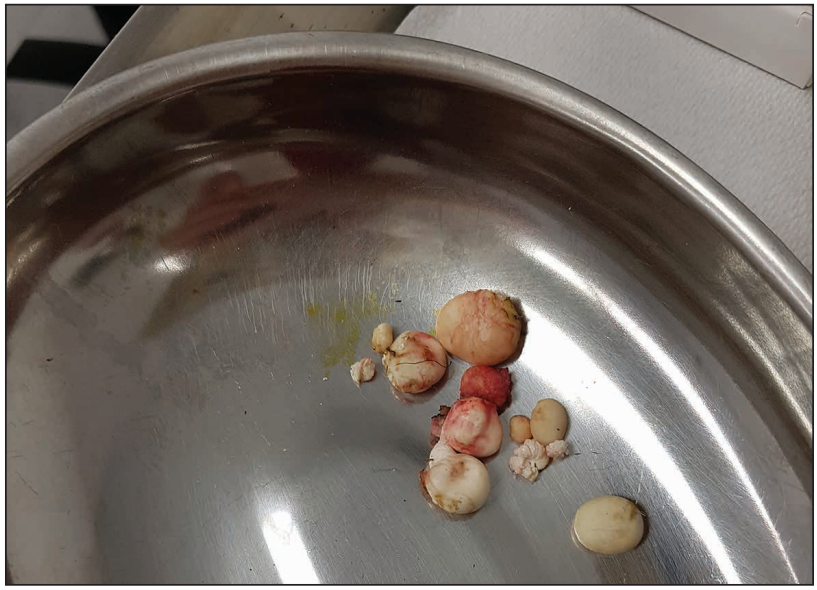

FIGURE 2. Macroscopic aspect of multiple excised nodules

Histopathological examination revealed collagen tissue that coats an area of amorphous material with multiple microcalcifications, surrounded by uninucleate and multinucleate macrophages. All tests conducted (determination of calcium and phosphorus levels, vitamin D level, parathyroid hormone level, specific antibodies for lupus erythematosus, markers for possible tumors) excluded metabolic, endocrine, autoimmune, neoplastic disorders.

Based on the anamnesis as well as the clinical and histopathological aspects, the diagnosis of idiopathic scrotal calcinosis was established. The treatment consisted in the surgical excision of the nodules with the removal of the hard, white-yellow, chalky content in two sessions. The medium and long-term postoperative evolution is favorable.

\section{DISCUSSIONS}

Idiopathic scrotal calcinosis (ISC) is a rare, benign condition, characterized by the appearance of solitary or multiple calcified nodules, located in the scrotum (8-10). Historically, it was first reported by Lewinski in 1883 and named as idiopathic scrotal calcinosis by Shapiro in $1970(4,8)$.

Despite of its benignity, the impact on the patient's quality of life can be very important. It appears mainly in men aged 20-40 years old and it is more common among dark colored skin patients, suggesting an ethnic susceptibility $(9,11)$.

Our patient, at 45 years of age, has had the initial nodules when he was around 20 years old, sup- porting the observation that it tends to develop in the second decade of life. Clinically, the nodules are firm, yellowish, with variable sizes $(0,5-3 \mathrm{~cm})$ and usually asymptomatic, sometimes they could be itchy or painful, located in the scrotum and the patient can present with chalk white discharge following an episode of infection. Also, the dermatological examination labelled the lesions as being similar to those described in the literature $(7,12,13)$.

ISC develops slowly, insidiously without any systemic disorder of the calcium/phosphorus metabolism. The diagnosis is often delayed, due to slow development and the intimate localization of the nodules. The pathogenesis of this condition still remains unclear and controversial $(1,3)$. Our patient had a similar record of a slow progression, little to no symptoms and the anamnesis was unable to pinpoint any of the usual trigger factors involved in ISC.

In all cases of calcinosis cutis, insoluble calcium compounds are deposited in the skin due to local and/or systemic factors. These calcium salts are mainly composed of hydroxyapatite crystals or amorphous calcium phosphate.

Metabolic and physical factors are absolutely necessary in the development of most cases of calcinosis. Ectopic calcification may occur in hypercalcemia and / or hyperphosphatemia when calcium-phosphate products exceed $70 \mathrm{mg} / \mathrm{dl}$, with no history of tissue alteration. These increased extracellular values may also increase intracellular levels, calcium phosphate nucleation and crystalline precipitation $(11,13,14)$.

The damaged tissue allows an influx of calcium ions, leading to increased intracellular calcium levels and secondary crystalline precipitation. Tissue damage can also lead to the formation of denatured proteins that preferentially bind phosphate. Then calcium reacts with bound phosphate ions, leading to the precipitation of calcium phosphate.

Numerous theories have been proposed concerning the origin of idiopathic scrotal calcinosis, such as the one where the nodules have emerged de novo without any triggering metabolic condition, infiltration of foreign body material or induced by trauma (9-11). In our patient's case no abnormalities in calcium metabolism were observed, the serum level of calcium, phosphate and parathyroid hormones was normal, which argues for the idiopathic etiology of calcinosis. Also, some research indicate that they are the result of dystrophic calcification of preexistent epidermal inclusion cysts (epidermoid, sebaceous, of the eccrine glands) $(9,10,12)$. 
Pabuççuoğlu et al. speculated that the degeneration and necrosis of dartos muscle played a prominent primary role in the etiopathogenesis of ISC following which dystrophic calcification occurred $(10,15,16)$.

Akinboro et al. are not in support of the hypothesis which suggests the origin of ISC from degeneration of dartos muscle, as the excision of the lesion showed that the encapsulated nodule occurred separately above the dartos fascia. They opined that the ISC could be the end stage of a single disease that started from multiple etiologies, reports from isolated and some large surgical anatomical reports support this view (10). Other theories suggest that ISC might be represented by the calcification of lymphangiomas, xanthomas, fibromas, teratomas, gonad blastomas $(4,13,17)$. However, the calcification of preexistent epidermal cysts is the most suggested theory by many authors as a possible pathogenesis.

For our case the differential diagnosis included tumoral formations that can lead to calcifications such as scrotal epidermoid cysts, sebum cystomatosis, benign tumors (lipomas, fibromas, angiokeratomas, circumscribed lymphangioma), testicular tumors (teratomas, gonad blastomas, Leydig cell tumors) $(8,13)$.

Paraclinical diagnosis can be supported by complex investigations; imaging studies such as CT scans will show calcifications in the scrotal wall but are usually not needed as diagnosis is mainly clinical and confirmed with histology of excised nodules (8).

In our case the histology sections revealed calcium deposition, and basophilic globules of varying sizes and shapes within the reticular dermis. There was an occasional scalloped appearance, with some of the smaller fragments surrounded by multinucleated giant cells and a sparse lymphocytic infiltrate. In between the globules, there were dense collagenous stroma and areas of fibrosis that were seen, and a fibrous capsule surrounded the entire lesion (Figure 3), as it is emphasized in other works $(8,10,18)$. Our patient also presented a histopathological examination suggestive for the diagnosis of idiopathic scrotal calcinosis.

A new developing technology to aid diagnosis is advanced vibrational spectroscopy. This diagnostic technique uses Fourier transform infrared (FTIR) or Raman spectroscopic analysis (RSA) to quickly identify the chemical composition of calcinosis skin lesions and can predict accurately the progression of the disease.

Even though the nodules are benign, the main reason why patients search medical advice remains

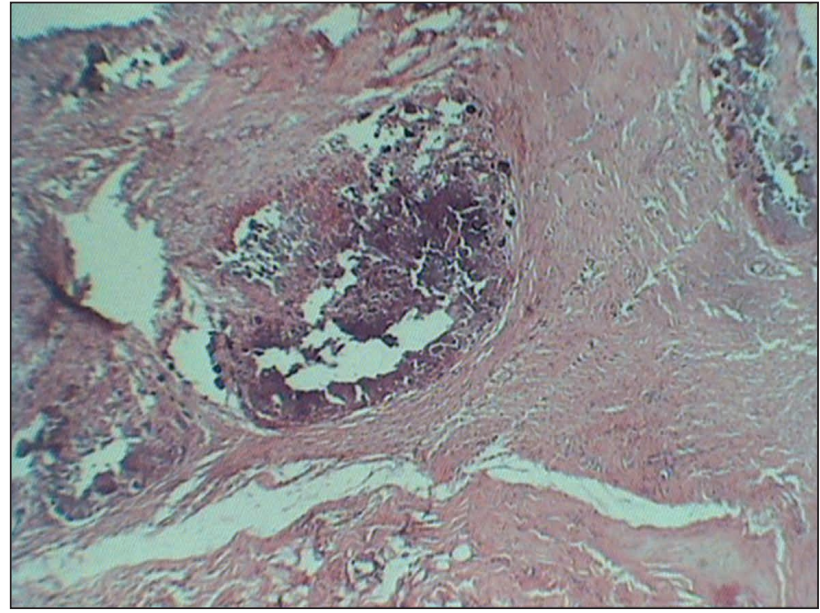

FIGURE 3. Histological aspect of a fibro collagenous structure delimiting an area of amorphous material with multiple calcifications (Col HE, Ob. 40x)

the aesthetic nature of the lesions and the impact of their sex life and self-esteem.

The treatment of choice is surgical excision. The extent of excision must include all nodules, even the smallest ones, to avoid rapid recurrence. The high risk of recurrence is to be considered.

In our patient's case, surgical excision of the nodules was performed with good outcome and satisfactory aesthetic results (Figure 4), improving the quality of life and boosting self-esteem. At 1 -year post-surgery, the patient did not have local recurrences, as it is described in literature $(1,6,8,14)$.

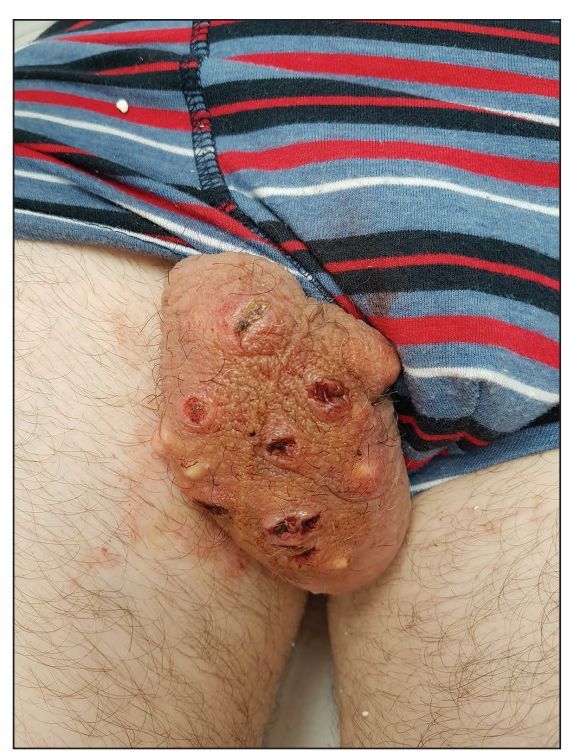

FIGURE 4. Postoperative aspect of the scrotum

\section{CONCLUSIONS}

Scrotal calcinosis is a rare and benign disease, with major impact on patient's life quality. The pathogenesis and basic origin remain controver- 
sial. The theory of the calcification of the preexistent epidermoid cysts appears to be most likely to believe. Complete surgical excision is the recommended treatment, it appears to provide good clinical outcome with low risk of recurrence. Impaired quality of life, self-esteem and fear of sexual dysfunction often led the patient to see a doctor and accept the surgical treatment.

The peculiarities of the presented case are the rarity of the condition and the long period of time before presentation from the onset of the disease (approximately 25 years) as this location, being in an intimate area, usually drives the patients much faster for medical advice. Therefore, clinicians

Conflict of interest: none declared

Financial support: none declared

\section{REFERENCES}

1. Dubey S, Sharma R, Maheshwari V. Scrotal calcinosis: idiopathic or dystrophic? Dermatol Online J. 2010;16(2):5.

2. Shivkumar VB, Gangane N, Kishore S, Sharma S. Cytologic features of idiopathic scrotal calcinosis. Acta Cytol. 2003;47(1):110-1.

3. Saad AG, Zaatari GS. Scrotal calcinosis: is it idiopathic? Urology. 2001;57(2):365.

4. Shapiro L, Platt N, Torres-Rodriguez VM. Idiopathic calcinosis of the scrotum. Arch Dermatol. 1970;102(2):199-204.

5. Yuyucu Karabulut Y, Kankaya D, Senel E, Dolek Y, Uslu A, Sertcelik A. Idiopathic scrotal calcinosis: the incorrect terminology of scrotal calcinosis. G Ital Dermatol Venereol. 2015;150(5):495-9.

6. Cecchi R, Giomi A. Idiopathic calcinosis cutis of the penis. Dermatology. 1999;198(2):174-5.

7. Duperrat B, Mascaro JM, Benoist M. Solitary nodular calcification of the skin (Winer type). Bull Soc Fr Dermatol Syphiligr. 1963 Jul-Aug;70:403-6.

8. Kyei MY, Djagbletey R, Abrahams AD, Mensah JE. Idiopathic Scrotal Calcinosis: A Case Report and Review of Postoperative Outcomes. Case Rep Urol. 2020;2020:8877695.

9. Heldhli H, Hermi A, Raboudi M, Ichaoui H, Khiari R, Ghozzi S, et al. Urology Case Reports. Urology Case Reports. Sept 2020;32.

10. Akinboro AO, Onilede DA, Babatunde TO, Oiwoh SO, Suleiman OA, Olabode OP. Idiopathic scrotal calcinosis: report of 2 cases, and review of pathogenesis and factors that determine patients acceptance of surgical treatment. Clin Cosmet Investig Dermatol. 2018;11:333-7. may not be familiar with this disease, leading to a significant delay in diagnosis to a refusal of the patient regarding the surgical treatment due to the intimate location of the nodules.

The presentation of this case brings into question the need for education and prevention campaigns for genital diseases in order to reduce the number of cases with delayed presentation and long periods of evolution that may render a surgical excision impossible.

\section{Acknowledgement}

All authors contributed in equal manner in drafting this case report.

11. Khallouk A, Yazami OE, Mellas S, Tazi MF, El Fassi J, Farih MH. Idiopathic scrotal calcinosis: a non-elucidated pathogenesis and its surgical treatment. Rev Urol. 2011;13(2):95-7.

12. Grenader T, Shavit L. Images in clinical medicine. Scrotal calcinosis. N Engl J Med. 2011 Aug 18;365(7):647.

13. Lei X, Liu B, Cheng Q, Wu J. Idiopathic scrotal calcinosis: report of two cases and review of literature. Int J Dermatol. 2012; 51(2):199-203.

14. Galezowski $A$, Sin $C$, Rothé-Thomas $F$, de la Bretèque MA, Bilan $P$, Sigal ML, Mahé E. Subcutaneous calcinosis, a late and severe complication of radiotherapy: Three cases. Annales de Dermatologie et de Vénéréologie 2015;142(12):761-6.

15. Pabuccuoglu U, Canda MS, Guray M, Kefi A, Canda E. The possible role of dartoic muscle degeneration in the pathogenesis of idiopathic scrotal calcinosis. Br J Dermatol. 2003;148(4):827-9.

16. Ruiz-Genao DP, Rios-Buceta L, Herrero L, Fraga J, Aragues M, Garcia-Diez A. Massive scrotal calcinosis. Dermatol Surg. 2002;28(8):745-7.

17. Noel W, Hersant B, Meningaud JP. One-staged surgical technique for scrotal calcinosis. Progres en urologie. 2016;26(3):176-80.

18. Carson DA. An infectious origin of extraskeletal calcification. Proceedings of the National Academy of Sciences of the United States of America. 1998;95(14):7846-7. 